\title{
Erratum
}

\section{The number of reducible hypersurfaces in a pencil}

\author{
Angelo Vistoli \\ Invent. math. 112, 247-262 (1993)
}

Due to a most unfortunate error, pages 249 and 250 were wrongly paginated and should have been placed between pages 258 and 259. We apologize for any inconvenience caused. 